\title{
OTIMIZAÇÃO DA HIDRÓLISE ÁCIDA DE AMIDO POR ULTRASSOM E REFLUXO
}

\author{
MARIA VERONICA FREITAS NASCIMENTO* \\ JUAREZ VICENTE ** \\ FILIPE OZORIO MACIEL DA SILVA*** \\ OTAVIO RAYMUNDO LÃ $\tilde{~}^{* * *}$
}

\begin{abstract}
A determinação do conteúdo de carboidratos é importante para a caracterização e para o controle de qualidade em diversos alimentos e bebidas. Um dos métodos mais utilizados para quantificação de açúcares em alimentos é o método de Lane-Eynon, em função do seu baixo custo, relativa simplicidade e por tratar-se de um método titulométrico. $\mathrm{O}$ objetivo deste artigo foi estudar alternativas viáveis para hidrólise de amido, utilizando ultrassom e refluxo, a fim de otimizar as variáveis tempo e concentração de amido que chegassem ao maior rendimento possível. Os resultados mostraram que o maior rendimento obtido foi utilizando refluxo, na concentração de $1 \%(\mathrm{~m} / \mathrm{m})$ no tempo de 20 minutos, com $10 \mathrm{ml}$ de $\mathrm{HCl}$. O uso do ultrassom não se mostrou eficiente.
\end{abstract}

PALAVRAS-CHAVE: MÉTODO DE LANE-EYNON; HIDRÓLISE ÁCIDA EM REFLUXO; HIDRÓLISE ÁCIDA EM ULTRASSOM.

* Discente do curso de Química Industrial, Departamento de Química, Instituto de Ciências Exatas, Universidade Federal Rural do Rio de Janeiro (UFRRJ), Seropédica, RJ (e-mail: veronica_quim@hotmail.com).

** Doutorando em Ciência e Tecnologia de Alimentos, Departamento de Tecnologia de Alimentos, Instituto de Tecnologia, Universidade Federal Rural do Rio de Janeiro (UFRRJ), Seropédica, RJ (e-mail: juarezvd@ gmail.com).

*** Discente do curso de Engenharia de Alimentos, Departamento de Tecnologia de Alimentos, Instituto de Tecnologia, Universidade Federal Rural do Rio de Janeiro (UFRRJ), Seropédica, RJ (e-mail: filipeozoriomaciel@hotmail.com).

**** Doutor em Agronomia - Ciências do Solo, docente, Departamento de Química, Instituto de Ciências Exatas, UFRRJ, Seropédica, RJ (e-mail: otaviola@ufrrj.br). 


\section{INTRODUÇÃO}

A determinação do conteúdo de carboidratos é importante para a caracterização e para o controle de qualidade em diversos alimentos e bebidas. Os carboidratos são as moléculas mais abundantes na natureza, constituídas principalmente por carbono, hidrogênio e oxigênio, podendo apresentar nitrogênio, fósforo ou enxofre em sua composição. Em alimentos, os carboidratos atuam basicamente como agentes de sabor (doçura), agentes de escurecimento (reações das carbonilas provenientes dos carboidratos) e agentes formadores de goma, influindo na textura dos alimentos.

Um dos métodos mais utilizados para quantificação de açúcares em alimentos é o de Lane-Eynon, em função do seu baixo custo, relativa simplicidade e por tratar-se de um método titulométrico. Entretanto, esse método é capaz de quantificar apenas açúcares redutores.

O procedimento recomendado pelo Ministério da Agricultura, Pecuária e Abastecimento (Mapa) para a hidrólise de açúcares não redutores envolve o uso de autoclave. A alta pressão combinada à alta temperatura utilizada na autoclave reduz o amido a açúcares redutores, como a glicose, porém açúcares menores já presentes na amostra, como glicose e sacarose, podem ser degradados. O uso do refluxo, bem como do ultrassom (sonoquímica), além de mais prático, pode proporcionar um melhor controle dos parâmetros utilizados para que essa hidrólise ocorra e se configurar como alternativa à utilização da autoclave.

O presente trabalho objetiva estudar o uso do ultrassom e do refluxo como alternativa de hidrólise de amido, em substituição ao uso da autoclave. Objetivamos determinar os parâmetros, o tempo e a concentração de amido ideais para a otimização da hidrólise na metodologia de quantificação de amido pelo método de Lane-Eynon.

\section{MATERIAL E MÉTODOS}

\subsection{MATERIAL}

Os reagentes utilizados neste experimento - amido, azul de metileno, sulfato de cobre penta-hidratado $\left(\mathrm{CuSO}_{4} \cdot 5 \mathrm{H}_{2} \mathrm{O}\right)$, tartarato duplo de sódio e potássio $\left(\mathrm{C}_{4} \mathrm{H}_{4} \mathrm{NaO}_{6} \cdot 4 \mathrm{H}_{2} \mathrm{O}\right)$, hidróxido de sódio $(\mathrm{NaOH})$, glicose padrão anidra $\left(\mathrm{C}_{6} \mathrm{H}_{12} \mathrm{O}_{6}\right)$ e ácido clorídrico $(\mathrm{HCl})$ - foram adquiridos da empresa Vetec (Brasil).

Foram utilizados: balança analítica (sensibilidade: $0,1 \mathrm{mg}$ ), medidor de $\mathrm{pH}$, agitador magnético e banho ultrassônico (Ultrasonic Cleaner, Odontobras, modelo 1440) a $40 \mathrm{kHz}$. A determinação de açúcares redutores foi realizada utilizando-se um determinador de açúcares redutores (Redutec - Modelo TE-088, Tecnal, Brasil).

\subsection{MÉTODOS}

As concentrações das soluções de amido e os tempos de hidrólise testados são apresentados na tabela 1. As letras representam os tempos utilizados $(A=20 \mathrm{~min}, B=25 \mathrm{~min}$ e $C=$ $30 \mathrm{~min}$ ), e os números, as concentrações de amido utilizadas, onde: 1 representa $0,5 \mathrm{~g}^{100 \mathrm{~mL}^{-1}, 2}$ $=1,0 \mathrm{~g} 100 \mathrm{~mL}^{-1}, 3=2,0 \mathrm{~g} 100 \mathrm{~mL}^{-1}$ e $4=5,0 \mathrm{~g} 100 \mathrm{~mL}^{-1}$ de amido.

As massas de amido (especificadas na tabela 1) foram pesadas $( \pm 0,1 \mathrm{mg})$ diretamente no erlenmeyer. Adicionaram-se $50 \mathrm{ml}$ de água destilada e $10 \mathrm{ml}$ de ácido clorídrico concentrado e prosseguiu-se para as condições de hidrólise em refluxo e ultrassom, como descrito a seguir. Todos os experimentos foram conduzidos em duplicata. 
TABELA 1 - TRATAMENTOS UTILIZADOS NOS LOTES DE AMIDO TESTADOS

\begin{tabular}{lcc}
\hline & Concentração de amido $\%(\mathbf{m} / \mathbf{m})$ & Tempo de hidrólise (minutos) \\
\hline Lote A1 & 0,5 & 20 \\
Lote B1 & 0,5 & 25 \\
Lote C1 & 0,5 & 30 \\
Lote A2 & 1,0 & 20 \\
Lote B2 & 1,0 & 25 \\
Lote C2 & 1,0 & 30 \\
Lote A3 & 2,0 & 20 \\
Lote B3 & 2,0 & 25 \\
Lote C3 & 2,0 & 30 \\
Lote A4 & 5,0 & 20 \\
Lote B4 & 5,0 & 25 \\
Lote C4 & 5,0 & 30 \\
\hline
\end{tabular}

\subsubsection{Hidrólise em ultrassom}

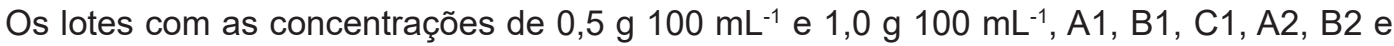
$\mathrm{C} 2$, foram colocados em um banho ultrassônico a $40 \mathrm{kHz}$ nos tempos predeterminados na tabela 1 para a hidrólise do amido.

\subsubsection{Hidrólise em refluxo}

Todos os lotes apresentados na tabela 1 foram pesados em um erlenmeyer de boca esmerilhada, conectados a um condensador e colocados sob refluxo (à temperatura de ebulição) nos tempos predeterminados.

\subsubsection{Hidrólise em autoclave}

Na melhor condição de rendimento obtida pela hidrólise por refluxo, utilizou-se o mesmo tempo e concentração para uma comparação com o método da autoclave (convencional), a qual foi realizada utilizando-se a pressão de $1 \mathrm{~atm}$ a $121^{\circ} \mathrm{C}$ por 20 minutos.

Após as hidrólises por autoclave, refluxo e ultrassom, as amostras foram resfriadas à temperatura ambiente $\left(25^{\circ} \mathrm{C}\right)$ e neutralizadas até $\mathrm{pH} 7,0 \pm 0,5 \mathrm{com}$ adição de $\mathrm{NaOH} \mathrm{0,1} \mathrm{mol.L-1}$. Em seguida, as soluções foram avolumadas para balão volumétrico de $100 \mathrm{ml}$ com água destilada.

\subsection{DETERMINAÇÃO DE AÇÚCARES REDUTORES}

A metodologia utilizada para a quantificação de açúcares redutores foi o método titulométrico de Lane-Eynon, recomendado pelo Ministério da Agricultura, Pecuária e Abastecimento (Mapa). Nesse método, o cobre (II) é reduzido a cobre (I), em meio alcalino, em uma reação com açúcares redutores (glicose).

\subsubsection{Preparo das soluções}

Solução de Fehling A: dissolver $34,639 \mathrm{~g}( \pm 1 \mathrm{mg})$ de sulfato de cobre penta-hidratado $\left(\mathrm{CuSO}_{4} \cdot 5 \mathrm{H}_{2} \mathrm{O}\right)$ p.a. em água destilada e diluir a $1.000 \mathrm{ml}$ em balão volumétrico.

Solução de Fehling B: dissolver $173 \mathrm{~g}$ de tartarato duplo de sódio e potássio $\left(\mathrm{C}_{4} \mathrm{H}_{4} \mathrm{NaO}_{6} \cdot 4 \mathrm{H}_{2} \mathrm{O}\right)$ p.a. e $125 \mathrm{~g}$ de hidróxido de sódio $(\mathrm{NaOH})$ p.a. em água. Diluir a $1.000 \mathrm{ml}$ em balão volumétrico. 
Solução-padrão de glicose $\left(\mathrm{C}_{\underline{6}} \underline{\mathrm{H}}_{12} \underline{\mathrm{O}}_{\underline{6}}\right)$ : secar glicose p.a., anidra, em estufa a $105^{\circ} \mathrm{C} \pm 2{ }^{\circ} \mathrm{C}$, por duas horas. Depois de resfriada em dessecador, pesar $0,5 \mathrm{~g}( \pm 0,1 \mathrm{mg})$ de glicose, solubilizar em água destilada e, em seguida, avolumar a $100 \mathrm{ml}$ em balão volumétrico.

\subsubsection{Padronização da solução de glicose e titulação das amostras}

Para determinação de açúcares redutores no Redutec ${ }^{\circledR}$, "licores" de reagentes são preparados em erlenmeyer de $125 \mathrm{ml}$ com a mistura de $10 \mathrm{ml}$ da solução de Fehling A, $10 \mathrm{ml}$ de Fehling $B$ e $40 \mathrm{ml}$ de água destilada. Com a caldeira aquecida, transfere-se o licor de Fehling para o compartimento reacional do Redutec ${ }^{\circledR}$, aguarda-se um minuto em ebulição e inicia-se a titulação com a solução-padrão de glicose anteriormente preparada ou com os açúcares redutores provenientes da amostra.

O licor Fehling é azulado devido à presença do íon $\mathrm{Cu}^{+2}$ na solução. Com a titulação com açúcares redutores, a coloração azulada da solução começa a diminuir de intensidade. Nesse momento, adicionam-se duas gotas de indicador azul de metileno e prossegue-se a titulação até a descoloração total da solução azul e aparecimento de um precipitado de coloração "vermelho-tijolo" $\left(\mathrm{Cu}_{2} \mathrm{O}\right)$, indicando o final da titulação. Essa titulação deverá ser feita sob ebulição, não podendo ultrapassar três minutos. $O$ resumo da análise é apresentado na figura 1.

Fehling A

\section{$34,639 \mathrm{~g} \mathrm{CuSO} 4.5 \mathrm{H}_{2} \mathrm{O}$ $1000 \mathrm{~mL}$ com $\mathrm{H}_{2} \mathrm{O}$ dest}

\section{Fehling B}

$173 \mathrm{~g}$ de tartarato duplo de sodio e potassio $\left(\mathrm{C}_{4} \mathrm{H}_{4} \mathrm{NaO}_{6} 4 \mathrm{H}_{2} \mathrm{O}\right)$

$+125 \mathrm{gNaOH}$

$1000 \mathrm{~mL}$ com $\mathrm{H}_{2} \mathrm{O}$ dest
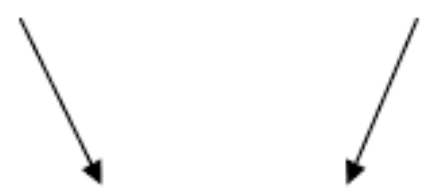

\section{Licor de Fehling}

$10,0 \mathrm{~mL}$ Fehling A

$10,0 \mathrm{~mL}$ Fehling B

$40,0 \mathrm{~mL}$ H2O dest

1-2 gotas indicador Azul de metileno $1 \%$
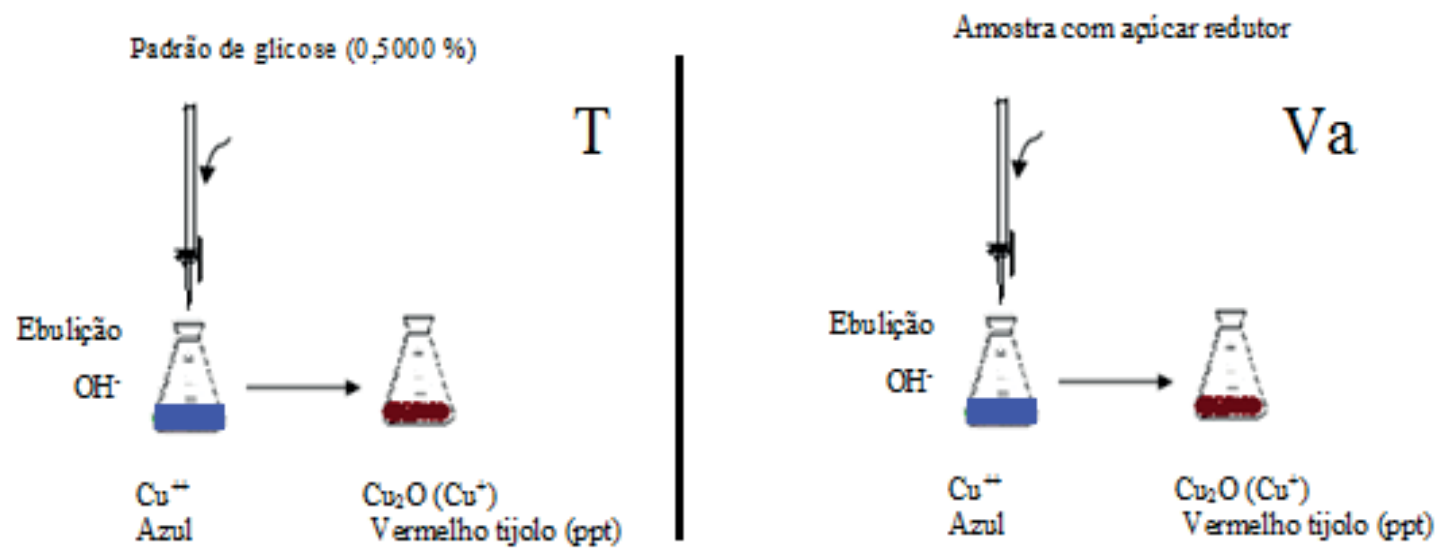

FIGURA 1 - ESQUEMA DA ANÁLISE DE AÇÚCARES REDUTORES E PADRONIZAÇÃO DA GLICOSE 


\subsubsection{Determinação do título (padronização) da solução do licor de Fehling}

Para o cálculo do título $(T)$, utiliza-se a equação 1, a seguir:

$$
T=\frac{V_{p} \cdot m_{p}}{d_{p}}
$$

Onde: $V_{p}=$ Volume gasto da solução-padrão de glicose na titulação

$m_{p}=$ massa de glicose $(\mathrm{g})$ utilizada no preparo da solução-padrão $(0,500 \mathrm{~g} \pm 1 \mathrm{mg})$

$d_{p}^{p}=$ volume final de preparo da solução-padrão de glicose $(100 \mathrm{ml})$.

Observação: diferentemente do que tradicionalmente se conhece em Química Analítica como título [utilizado para designar a concentração ( $\mathrm{g} / \mathrm{l})$ de um analito], a nomenclatura "título" é atribuída pelo Mapa à massa de glicose necessária à redução do $\mathrm{Cu}^{+2}$ (presente na solução de Fehling A) a $\mathrm{Cu}^{+1}\left(\mathrm{Cu}_{2} \mathrm{O}\right)$. O objetivo dessa titulação é "padronizar" a solução de Fehling a partir de uma massa conhecida de açúcar redutor (glicose).

\subsection{TRATAMENTO ESTATÍSTICO DOS DADOS}

Os resultados de cada lote foram expressos pela média \pm desvio-padrão. ANOVA (análise de variância) foi aplicada ao conjunto de tratamentos, ao nível de significância de $5 \%$ ( $\rho$ $<0,05$ ). Havendo diferença significativa em pelo menos um dos tratamentos, foi aplicado o teste de Tukey para médias.

\section{RESULTADOS E DISCUSSÃO}

\subsection{RESULTADOS DOS TRATAMENTOS ESTUDADOS}

\subsubsection{Utilização da hidrólise do amido em autoclave}

A hidrólise do amido utilizando a autoclave é rotina no laboratório. O rendimento situa-se ao redor de $80 \%$. Sendo assim, o do uso da autoclave foi utilizado apenas com o lote A2, em que as condições de massa de amido $(1,0 \mathrm{~g})$ e tempo de hidrólise $(20 \mathrm{~min})$ foram as de maior rendimento $(99,4 \%)$ pelo método do refluxo. O rendimento obtido para o lote A2, com autoclave, foi de $80,9 \% \pm 0,7 \%(X \pm s)$. Pode-se inferir que as condições de temperatura e pressão utilizadas na autoclave para reduzir amido em glicose são severas a ponto de provocar a degradação de parte da glicose produzida.

\subsubsection{Utilização da hidrólise do amido em refluxo}

A tabela 2 apresenta os resultados médios obtidos com a hidrólise do amido utilizando o refluxo, com os respectivos desvios-padrão e classificação algébrica dados pela análise de variância (ANOVA).

Aplicando-se a ANOVA aos dados constantes na tabela 2, observou-se o valor $p$ abaixo de 0,05 . Com isso, seguiu-se a análise com o cálculo de diferença mínima significativa (dms) através do teste de Tukey (Eq. 2) dos lotes estudados.

O dms calculado foi igual a 3,1. As médias dos tratamentos que apresentam a mesma classificação (letras iguais) não diferem estatisticamente.

O tratamento $\mathrm{A} 2$, com tempo de hidrólise em refluxo por 20 minutos na concentração de $1 \%$ $(\mathrm{g} / 100 \mathrm{~g})$ de amido, não diferiu estatisticamente do tratamento B2 (1 g-25'), porém esse tratamento (A2) difere de todos os outros. 


\begin{tabular}{llll}
\hline & Média & \pm & Desvio-padrão \\
\hline \hline Lote A1 & $83,5^{\mathrm{f}}$ & \pm & 0,3 \\
Lote B1 & $86,3^{\mathrm{f}}$ & \pm & 0,8 \\
Lote C1 & $86,0^{\mathrm{f}}$ & \pm & 1,8 \\
Lote A2 & $99,4^{\mathrm{a}}$ & \pm & 0,5 \\
Lote B2 & $96,4^{\text {ab }}$ & \pm & 0,5 \\
Lote C2 & $92,5^{\text {cde }}$ & \pm & 0,6 \\
Lote A3 & $92,5^{\text {cde }}$ & \pm & 0,5 \\
Lote B3 & $95,1^{\mathrm{bc}}$ & \pm & 1,0 \\
Lote C3 & $95,7^{\mathrm{b}}$ & \pm & 0,8 \\
Lote A4 & $94,6^{\mathrm{bcd}}$ & \pm & 0,3 \\
Lote B4 & $91,7^{\text {de }}$ & \pm & 0,3 \\
Lote C4 & $91,1^{\mathrm{e}}$ & \pm & 0,6 \\
\hline
\end{tabular}

Os lotes que utilizaram a concentração de $0,5 \%$, independentemente do tempo de refluxo, foram os de menor recuperação. Como a concentração de amido é a menor entre as concentrações estudadas, pode indicar que ocorreu uma maior degradação dos açúcares redutores durante a hidrólise, sendo possível que a quantidade de ácido clorídrico $(10 \mathrm{ml})$ adicionada para hidrólise tenha sido excessiva para a concentração de amido estudada. Nesse caso, pode ter havido a redução e uma maior degradação da glicose produzida.

Nos lotes em que se utilizou a concentração de amido igual a 1\%, o aumento no tempo de refluxo influenciou negativamente a recuperação do amido. Já na concentração de amido a $2 \%$, o tempo parece tender a influenciar de forma contrária: quanto maior o tempo de refluxo, maior a recuperação.

Embora não se tenha feito o experimento completo com os vários tratamentos também utilizados para o método com autoclave, parece que os tratamentos (A2 e B2) foram mais eficientes na hidrólise de amido do que na autoclave.

\subsubsection{Utilização da hidrólise do amido com ultrassom}

Testou-se a possibilidade de hidrolisar o amido utilizando o banho de ultrassom a $40 \mathrm{kHz}$, nas mesmas concentrações e tempos utilizados na hidrólise por refluxo. Foram testados os lotes 1 ( $A, B$ e $C)$ e 2 ( $A, B, C)$.

Foi observado que os resultados obtidos nesses dois lotes, através do rendimento da conversão do amido em açúcares redutores, foram menores que $20 \%$. Pelos resultados encontrados (não mostrados), o uso do ultrassom como forma de substituir a autoclave na hidrólise do amido foi desconsiderado. Nos lotes subsequentes, previa-se a utilização de concentrações de amido ainda maiores. A partir de então, foi descontinuado o uso do ultrassom no experimento. 


\section{CONCLUSÃO}

A hidrólise realizada em refluxo configura-se como uma ótima alternativa ao uso da autoclave para quantificação de amido pelo método de Lane-Eynon (99,4\%). A concentração de $1 \%$ de amido, durante o tempo de 20 minutos com $10 \mathrm{ml} \mathrm{HCl}$, foi o parâmetro ideal. A hidrólise realizada em ultrassom não se mostrou eficiente para esse método.

\section{ABSTRACT}

\section{ACID HYDROLYSIS OPTIMIZATION OF STARCH BY LANE-EYNON METHOD.}

The carbohydrate content determination is important for the characterization and quality control in various foods and beverages. One of the most used methods for the quantification of sugars in foods is the Lane-Eynon method, due to its low cost, relative simplicity and because it is a titration method. The aim of this paper was to study viable alternatives to hydrolyze starch, using ultrasound and reflux in order to optimize the variables of time and concentration of starch that reached the best possible performance. The results showed that the highest yield was obtained using reflux at a concentration of $1 \%(\mathrm{w} / \mathrm{w})$ at time $20 \mathrm{~min}$ and $10 \mathrm{ml} \mathrm{HCl}$. The use of ultrasound was not efficient.

KEY-WORDS: LANE-EYNON METHOD, ACID REFLUX HYDROLYSIS, ACID HYDROLYSIS ULTRASOUND.

\section{REFERÊNCIAS}

1. ALLINGER, N. L. et al,.Hidratos de Carbono. Química Orgánica. Segunda edición. Barcelona: Editorial Reverté, As, 1991. Cap 25, p 959-997.

2. BRASIL. Ministério da Agricultura. Instrução Normativa $n^{\circ} 20$ de 21 de julho de 1999. Dispõe sobre os métodos analíticos físico-químicos para controle de produtos cárneos e seus ingredientes - sal e salmoura. Diário Oficial da República Federativa do Brasil, Brasília, 27 jul. 1999.

3. FRANCISCO Jr., W. E. Carboidratos: Estrutura, Propriedades e Funções. Química Nova na Escola - Conceitos Científicos em Destaque, Ararraquara, n 29. Ago 2008.

4. HODGE, J. E.; OSMAN, E. M. Carbohydrates.In: FENNEMA, O. R. Principles of Food Science - Food Chemistry. Part I. V4. Madison: Marcel Dekker, Inc, 1976. Cap 3, p 41-138.

5. INSTITUTO ADOLFO LUTZ (São Paulo - Brasil). Métodos físico-químicos para análise de alimentos: normas analíticas do Instituto Adolfo Lutz. $5^{\text {a }}$ ed. Brasília (DF): ANVISA; 2009.

6. MACHADO, F. L. C.; CAMPOS, G. \& SOUZA, M. T. G. Comparação entre os métodos de Lane-Eynon e polarimétrico para determinação de amido em farinha de mandioca. Rev Inst Adolfo Lutz, 68(1):155-9, 2009.

7. MECOZZI, M.; AMICI, M.; PIETRANTONIO, E.; \& ACQUISTUCCI, R. Ultrasound-assisted analysis of total carbohydrates in environmental and food samples. Ultrasonics Sonochemistry, 6, 133-139, 1999

8. MECOZZI, M.; ACQUISTUCCI, R.; AMICI, M.; \& CARDARILLI, D. Improvement of an ultrasound assisted method for the analysis of total carbohydrate in environmental and food samples. Ultrasonics Sonochemistry, 9, 219-223, 2002.

9. MOORE, D. S. The Basic Practice of Statistics, 5th ed. WH Freeman, NY. 2010.

10. VICENTE, J.; NEVES, M. A.; MELEIRO, L. A. C. Estimativa de incerteza de medição associada à análise de açúcares redutores pelo método de Lane-Eynon. B. CEPPA, Curitiba, v. 32, n. 1, p. 69-82, 2014. 
AGRADECIMENTOS

Os autores agradecem ao LAAB-Rural pela estrutura oferecida na realização deste estudo. 